\title{
Nose-to-Brain Delivery of Cancer-Targeting Paclitaxel-loaded Nanoparticles Potentiates Anti-Tumor Effects in Malignant Glioblastoma
}

Irfan Ullah ${ }^{1,2 \#}$, Kunho Chung ${ }^{1 \#}$, Sumin Bae ${ }^{1}$, Yan $\mathrm{Li}^{1,3}$, Chunggu Kim ${ }^{1}$, Boyoung Choi ${ }^{1,4}$, Hye Yeong Nam ${ }^{4}$, Sun Hwa Kim ${ }^{5}$, Chae-Ok Yun ${ }^{1}$, Kuen Yong Lee ${ }^{1}$, Priti Kumar ${ }^{2, *}$ and Sang-Kyung Lee $^{1,2, *}$

${ }^{1}$ Department of Bioengineering and Institute of Nanoscience and Technology, Hanyang University, Seoul, Korea, ${ }^{2}$ Department of Internal Medicine, Section of Infectious Diseases, Yale University School of Medicine, New Haven, USA, ${ }^{3}$ National Cancer Center, Gyeonggi-do, Korea, ${ }^{4}$ Samyang Biopharmaceuticals Co., Seoul, Korea, ${ }^{5}$ Biomedical Research Institute, Korea Institute of Science and Technology, Seoul, Korea.

*Correspondence should be addressed to P.K (priti.kumar@yale.edu) or S.K.L. (sangkyunglee@hanyang.ac.kr)

\# These authors contributed equally

Running title: Intranasal delivery of brain cancer-targeting paclitaxel-loaded nanoparticles for glioblastoma therapy 
Supplementary Data

Supplementary Figure 1

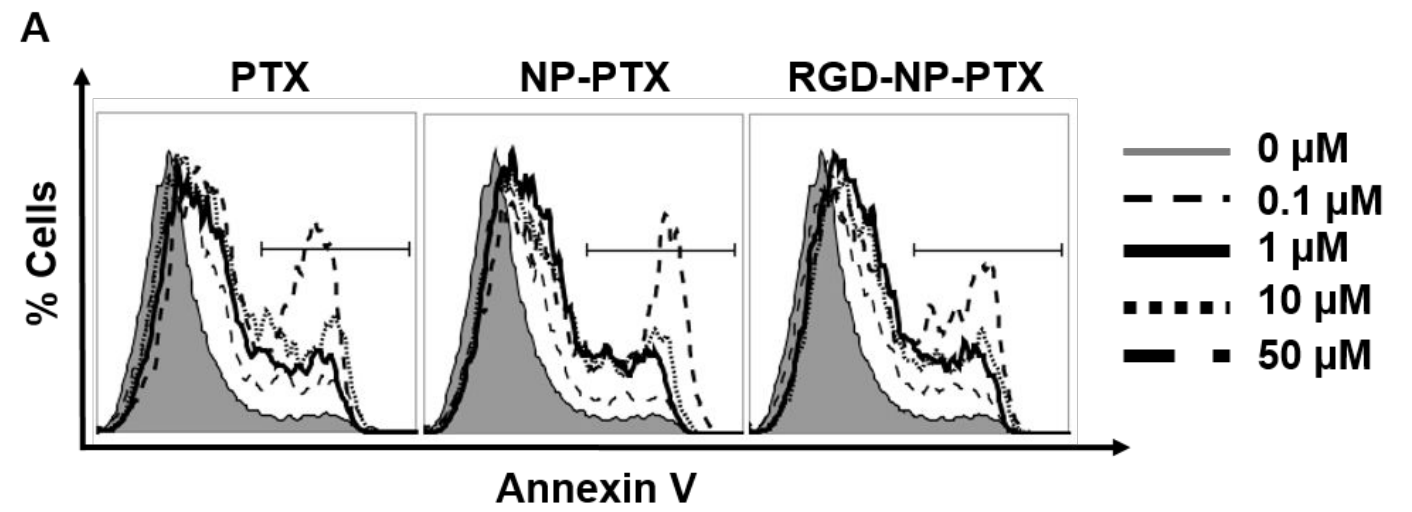

B

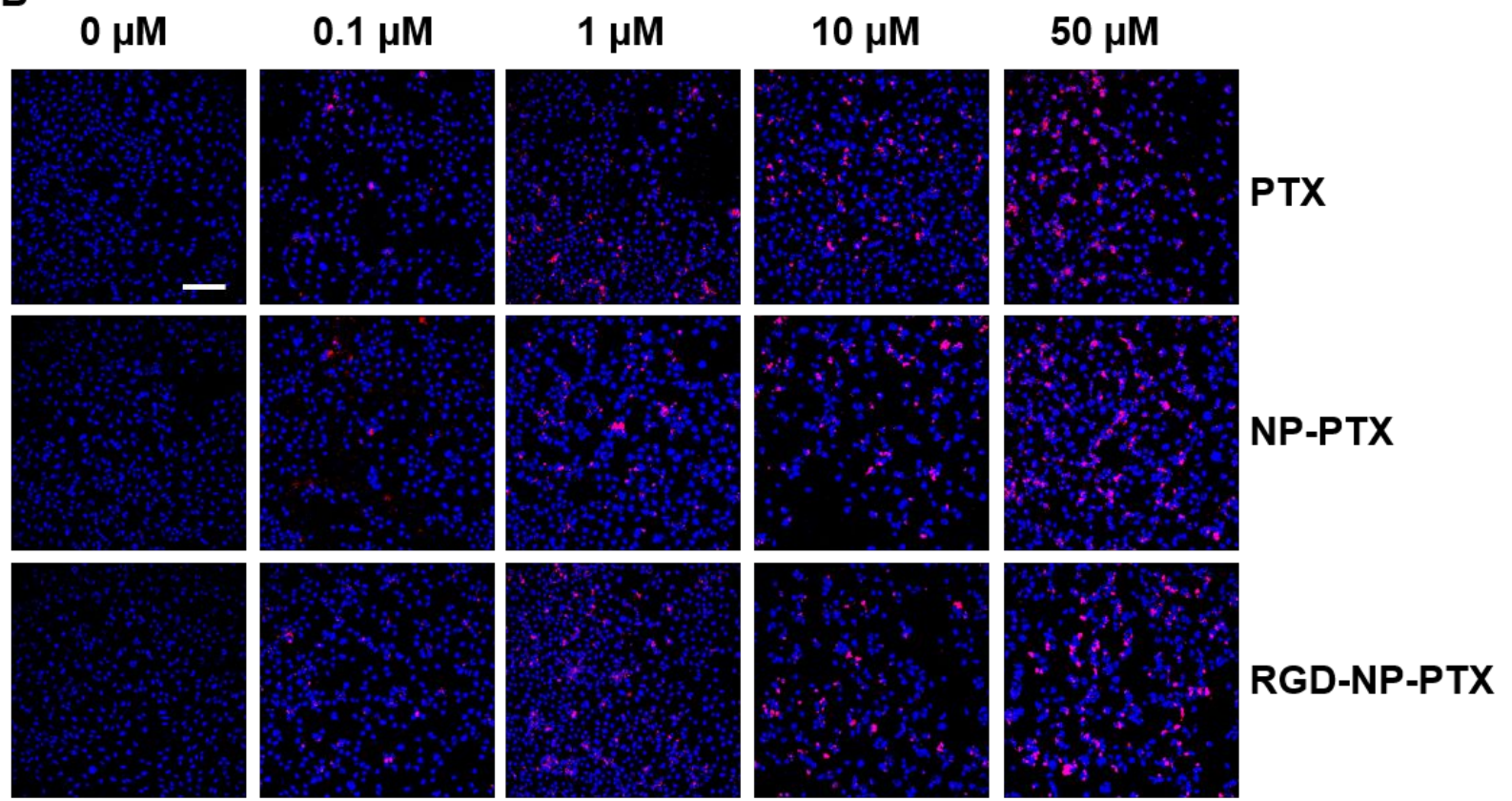

Magnification 200x 
Supplementary Figure 2

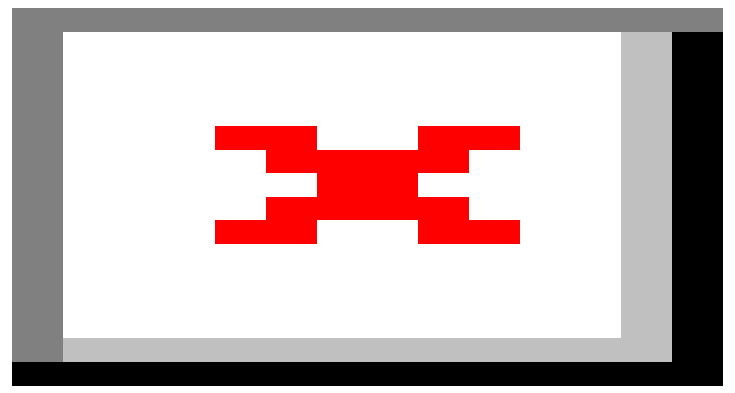


Supplementary Figure 3
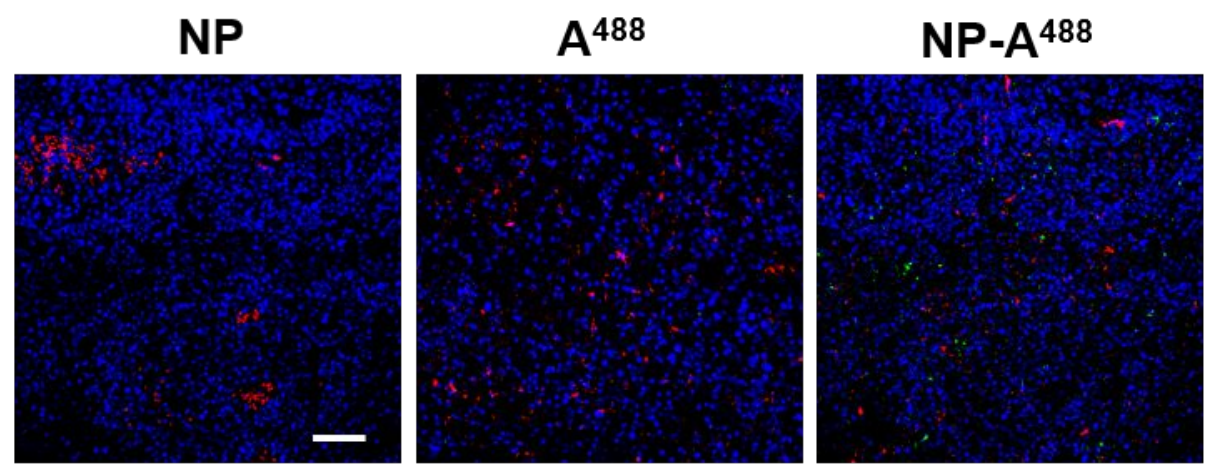

RGD-NP-A ${ }^{488}$

Magnification 400x

DAPI, Av $\beta 3, A^{488}$

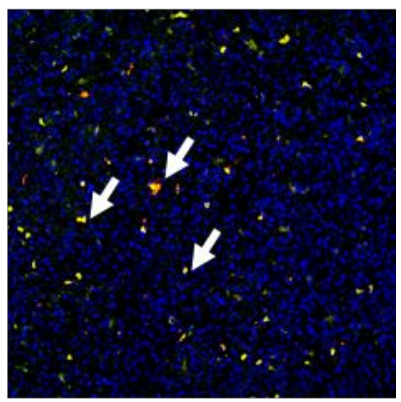


Supplementary Figure 4

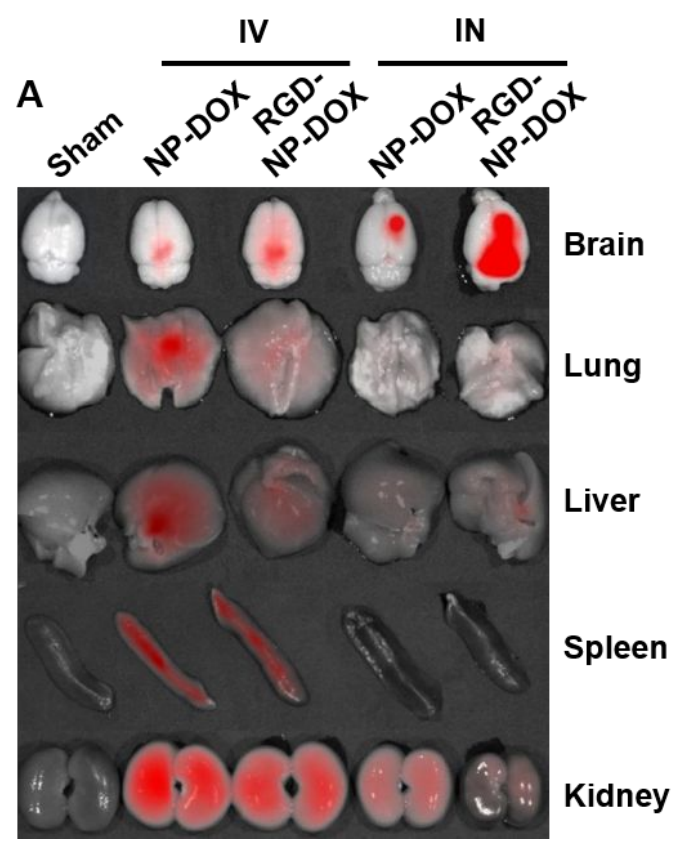

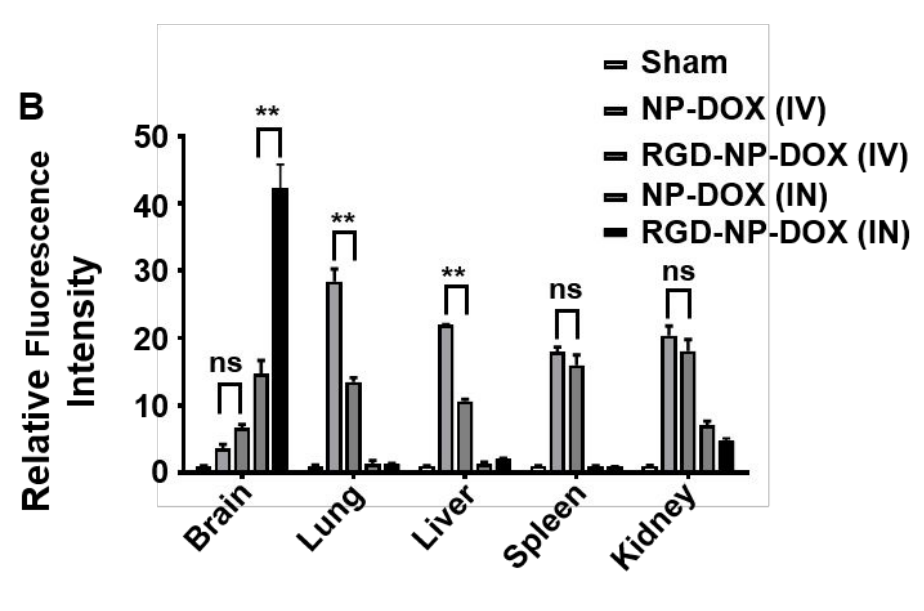


Supplementary Figure 5
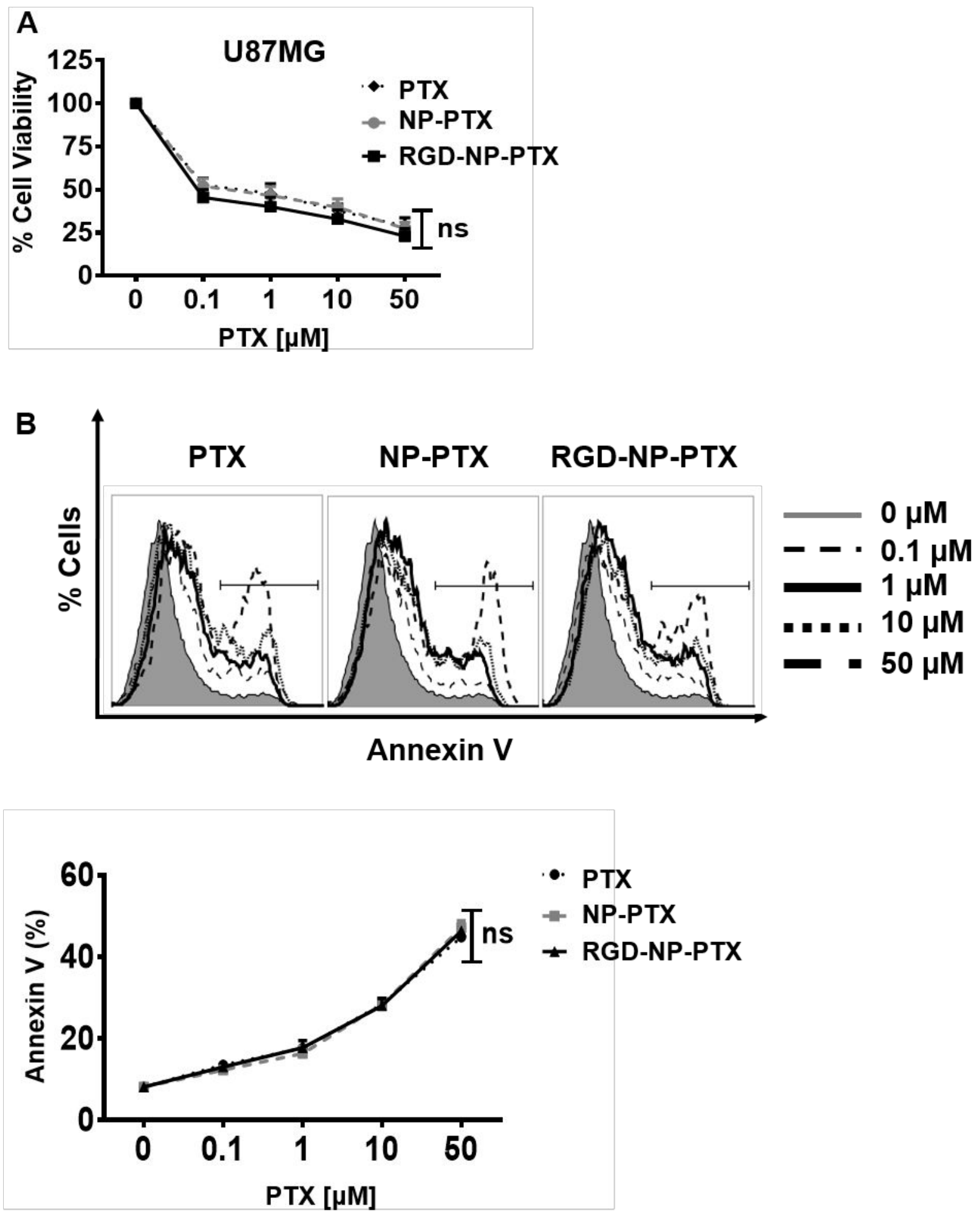


\section{Supplementary Figure 1}

(A) Flow cytometric analysis of apoptosis in C6 glioma cells $24 \mathrm{~h}$ post-treatment with PTX, NPPTX, or RGD-NP-PTX. Representative histogram are shown. (B) PTX-loaded NPs induced apoptosis in C6 glioma cells $24 \mathrm{~h}$ post-treatment. The representative confocal laser scanning images showing TUNEL-positive cells (red) and Hoechst-stained nuclei (blue) of equivalent PTXtreated C6 cells at the indicated concentration. Scale bar represents $50 \mu \mathrm{m}$ and the magnification is $200 x$.

\section{Supplementary Figure 2}

Uptake kinetics of rho-B loaded-NPs. Flow cytometric analysis rhoB delivery in C6 glioma cells at indicated time post-treatment with NP-rhoB or RGD-NP-rhoB. Representative histograms are shown (left) and cumulative data showing mean fluorescent intensities (MFI) (right). Data represents the mean $\pm \mathrm{SD}$ from triplicate assay. $* * P<0.01$, $* P<0.1$, and ns not-significant.

\section{Supplementary Figure 3}

Confocal microscopy images of brain sections. The representative images are shown the colocalization of RGD-NP-A ${ }^{488}$ (green), Integrin $\beta 3$ (red) and Hoechst-stained nuclei (blue) in the glioblastoma region. The scale bar represents $50 \mu \mathrm{m}$ and magnification is $400 \times$.

\section{Supplementary Figure 4}

(A) Biodistribution of IV or IN delivered NPs $(n=3)$. Brain, lung, liver, spleen and kidney were examined for the presence of DOX at $48 \mathrm{hr}$ post-inoculation of NP-DOX, RGD-NP-DOX. PBS (Sham) was used as a negative control. Representative images (A) and cumulative data (B) show the relative fluorescence intensities from the indicated organ measured in arbitrary pixel values 
when compared to the normalized sham. Data represents the mean $\pm \mathrm{SD}$. $* * P<0.01$, and ns notsignificant.

\section{Supplementary Figure 5}

(A) The cytotoxic effect measured by the CCK-8 assay $24 \mathrm{~h}$ post treatment of U87MG glioma cells with indicated equivalent PTX concentration. (B) Flow cytometric analysis of apoptosis in U87MG glioma cells 24 h post-treatment with PTX, NP-PTX, or RGD-NP-PTX. Representative histogram (B, upper) and cumulative data depicting \% annexin V (B, lower) are shown. ns- notsignificant. 